\title{
GEVREY REGULARITY OF CERTAIN SOLUTIONS TO THE CAHN-HILLIARD EQUATION WITH ROUGH INITIAL DATA*
}

\author{
DAVID SWANSON ${ }^{\dagger}$
}

Abstract. In this article we consider the problem of local Gevrey regularity of periodic solutions to the Cahn-Hilliard equation with initial data in a space of distributions. The method presented in this paper is based on the analysis of the Navier-Stokes system presented in [2] and makes use of a convolution inequality due to Kerman [3].

Key words. Cahn-Hilliard equation, Gevrey regularity.

AMS subject classifications. 35Q35, 35B65, 35K35.

1. Introduction. The Cahn-Hilliard equation we shall study is

$$
\begin{aligned}
& u_{t}=-\Delta^{2} u-\alpha \Delta u+\beta \Delta\left(u^{3}\right), x \in \Omega=[0, L]^{n}, t>0, \\
& u(x, 0)=u_{0}(x), x \in \Omega,
\end{aligned}
$$

where $\alpha \geq 0$, and $\beta>0$ and $u$ satisfies a periodic boundary condition on $\partial \Omega$. We assume for simplicity that $L=2 \pi$.

We let $\phi_{k}, k \in \mathbb{Z}^{n}$, denote the $k^{\text {th }}$ Fourier mode of a space periodic function $\phi$, so that $\phi$ may be identified with its Fourier series

$$
\phi \sim \sum_{k \in \mathbb{Z}^{n}} \phi_{k} e^{\imath k x} .
$$

The sequence of Fourier coefficients of $\phi$ is denoted by $\vec{\phi}=\left\{\phi_{k}\right\}$. Equation (1.1) may be cast as an infinite dimensional complex-valued dynamical system taking the form

$$
\frac{d}{d t} u_{k}=-|k|^{4} u_{k}+\alpha|k|^{2} u_{k}-\beta B[\vec{u}]_{k}, \quad k \in \mathbb{Z}^{n},
$$

where $B[\vec{u}]=B[\vec{u}, \vec{u}, \vec{u}]$ is a trilinear term satisfying

$$
B[\vec{u}, \vec{v}, \vec{w}]_{k}=|k|^{2} \sum_{j} \sum_{h} u_{j} v_{h} w_{k-j-h}
$$

We denote by $A$ the operator $\vec{v} \mapsto A \vec{v}$ given by $(A \vec{v})_{k}=|k|^{2} v_{k}$, so that the system (1.1)-(1.2) takes the form

$$
\begin{aligned}
& \vec{u}_{t}=-A^{2} \vec{u}+\alpha A \vec{u}-\beta B[\vec{u}] . \\
& \vec{u}(0)=\vec{u}_{0} .
\end{aligned}
$$

\section{Main result.}

Definition 2.1. We denote by $V$ the vector space of all complex valued sequences $\vec{v}=\left\{v_{k}\right\}_{k \in \mathbb{Z}^{n}}$ defined on $\mathbb{Z}^{n}$. Given $\vec{v} \in V, \lambda \geq 0, \theta \in \mathbb{R}$, and $1 \leq p<\infty$ we define

$$
\|\vec{v}\|_{\lambda, \theta, p}=\left(\sum_{k \in \mathbb{Z}^{n}} e^{\lambda p|k|}(1+|k|)^{\theta p}\left|v_{k}\right|^{p}\right)^{1 / p}
$$

\footnotetext{
*Received January 19, 2012; accepted for publication June 11, 2012.

${ }^{\dagger}$ Department of Mathematics, 328 Natural Science Building, University of Louisville, Louisville KY 40292, USA (david.swanson@louisville.edu).
} 
and

$$
V_{\lambda, \theta, p}=V \cap\left\{\vec{v}:\|\vec{v}\|_{\lambda, \theta, p}<\infty\right\} .
$$

In case $\lambda=0$ we write $\|\vec{v}\|_{\theta, p}$ and $V_{\theta, p}$ in place of $\|\vec{v}\|_{0, \theta, p}$ and $V_{0, \theta, p}$, respectively. $V_{\lambda, \theta, p}$ is a Banach space with norm $\|\cdot\|_{\lambda, \theta, p}$. We denote by $\dot{V}$ the vector space

$$
\dot{V}=V \cap\left\{\vec{v}: v_{0}=0\right\}
$$

and define

$$
\dot{V}_{\lambda, \theta, p}=\dot{V} \cap V_{\lambda, \theta, p}
$$

For any $\vec{v} \in \dot{V}_{\lambda, \theta, p}$ the norms

$$
\|\vec{v}\|_{\lambda, \theta, p} \quad \text { and } \quad\left(\sum_{k \in \mathbb{Z}^{n}} e^{\lambda p|k|}|k|^{\theta p}\left|v_{k}\right|^{p}\right)^{1 / p}
$$

are equivalent.

Definition 2.2. A mild solution to equation (1.1) with initial data in $\dot{V}_{\theta, p}$ is a map $\vec{u}(\cdot) \in C\left([0, T] ; \dot{V}_{\theta, p}\right)$ satisfying

$$
\vec{u}(t)=e^{-t\left(A^{2}-\alpha A\right)} \vec{u}_{0}-\beta \int_{0}^{t} e^{-(t-s)\left(A^{2}-\alpha A\right)} B[\vec{u}(s)] d s, \quad 0 \leq t \leq T .
$$

for some $T>0$. A mild solution $\vec{u}$ is said to be Gevrey regular if, in addition to satisfying (2.1), there exists $\lambda>0$ with the property that

$$
\sup _{0 \leq t \leq T}\|\vec{u}(t)\|_{\lambda t, \theta, p}<\infty
$$

Theorem 2.3. Let $1<p<\infty$, let $\theta=\frac{n}{p^{\prime}}-1$ where $p^{\prime}=p /(p-1)$, and let $\lambda>0$. Then

1. For any $T>0$ there exists a solution $\vec{u} \in C\left([0, T] ; V_{\theta, p}\right)$ to (2.1) satisfying (2.2) provided that $\beta>0$ is sufficiently small.

2. For any $\beta>0$ there exist $T>0$ and a solution $\vec{u} \in C\left([0, T] ; V_{\theta, p}\right)$ to (2.1) satisfying (2.2).

It is well-known that weak solutions to (1.1) exist for all times $T>0$ provided that the initial data belongs to $L^{2}(\Omega)$, cf. [5, Ch. III, Theorem 4.2]. Solutions in the sense of (2.1) are known to exist for initial data in $V_{2,2}$, cf. [4, Lemma 55.3]. In case $n=1,2,3$ the initial data spaces allowed in Theorem 2.3 allow for rougher initial data than the classical existence theory. Recently Biswas and Bae [1] obtained general estimates which when $p=2$ yield Theorem 2.3 as a special case. The result presented in this paper is valid for general $p$ and is based on the analysis of convolution inequalities rather than methods of harmonic analysis.

To prove Theorem 2.3 we let $T>0$ and fix a parameter $\gamma>0$ satisfying

$$
\min \left\{1-\frac{2 n}{3 p^{\prime}}, 1-\frac{n}{3 p^{\prime}}-\frac{n}{3 p}\right\}<\gamma<1
$$


where $p^{\prime}$ denotes the Hölder conjugate of $p$. We employ the path space

$E=C\left([0, T] ; \dot{V}_{\theta, p}\right) \cap\left\{\vec{u}: \sup _{0 \leq t \leq T}\|\vec{u}(t)\|_{\lambda t, \theta, p}<\infty\right\} \cap\left\{\vec{u}: \sup _{0<t \leq T} t^{\frac{\gamma}{4}}\|\vec{u}(t)\|_{\lambda t, \theta+\gamma, p}<\infty\right\}$

with norm

$$
\|\vec{u}(\cdot)\|_{E}=\sup _{0 \leq t \leq T}\|\vec{u}(t)\|_{\lambda t, \theta, p}+\sup _{0<t \leq T} t^{\frac{1}{4}}\|\vec{u}(t)\|_{\lambda t, \theta+1, p}
$$

We will obtain a solution to (2.1), (2.2) by showing that for $\vec{u}_{0} \in V_{\theta, p}$ the map $t \mapsto e^{-t\left(A^{2}-\alpha A\right)} \vec{u}_{0}$ belongs to $E$ and that the trilinear functional $S$ on $E \times E \times E$ defined by

$$
S(\vec{u}(t), \vec{v}(t), \vec{w}(t))=\beta \int_{0}^{t} e^{-(t-s)\left(A^{2}-\alpha A\right)} B[\vec{u}(s), \vec{v}(s), \vec{w}(s)] d s
$$

is bounded from $E \times E \times E$ to $E$.

3. Estimate on the linear term. Define

$$
\kappa=\kappa_{\alpha, \lambda}=-\min \left\{\frac{1}{2} x^{4}-\alpha x^{2}-\lambda x: x \in \mathbb{R}\right\} .
$$

We will show that for $\vec{u}_{0} \in \dot{V}_{\theta, p}$ the map $t \mapsto e^{-t\left(A^{2}-\alpha A\right)} \vec{u}_{0}$ belongs to $E$. We make repeated use of the elementary inequality

$$
e^{-a t x^{4}} x^{b} \leq C_{a, b} t^{-\frac{b}{4}}, \quad a, b, t, x>0 .
$$

Proposition 3.1. If $\vec{v} \in \dot{V}_{\theta, p}$, then $t \mapsto e^{-t\left(A^{2}-\alpha A\right)} \vec{v}$ belongs to $E$.

Proof. Let $\vec{v} \in \dot{V}_{\theta, p}$. Equation (3.1) implies

$$
\left\|e^{-t\left(A^{2}-\alpha A\right)} \vec{v}\right\|_{\lambda t, \theta, p}^{p}=\sum_{k} e^{\lambda p t|k|} e^{-p t\left(|k|^{4}-\alpha|k|^{2}\right)}|k|^{\theta p}\left|v_{k}\right|^{p} \leq e^{p \kappa t} \sum_{k} e^{-\frac{p}{2} t|k|^{4}}|k|^{\theta p}\left|v_{k}\right|^{p}
$$

so that

$$
\left\|e^{-t\left(A^{2}-\alpha A\right)} \vec{v}\right\|_{\theta, p} \leq e^{\kappa t}\|\vec{v}\|_{\theta, p}
$$

for all $0 \leq t \leq T$. Similarly, equations (3.1) and (3.2) imply

$$
\left\|e^{-t\left(A^{2}-\alpha A\right)} \vec{v}\right\|_{\lambda t, \theta+\gamma, p}^{p} \leq e^{p \kappa t} \sum_{k} e^{-\frac{p}{2} t|k|^{4}}|k|^{\theta+\gamma}\left|v_{k}\right|^{p} \leq C_{\gamma, p} e^{p \kappa t} t^{-\frac{p \gamma}{4}} \sum_{k}|k|^{\theta p}\left|v_{k}\right|^{p}
$$

so that

$$
t^{\frac{\gamma}{4}}\left\|e^{-t\left(A^{2}-\alpha A\right)} \vec{v}\right\|_{\theta+\gamma, p} \leq C_{\gamma, p} e^{\kappa t}\|\vec{v}\|_{\theta, p}
$$

for all $0<t \leq T$. Finally we may combine the estimates (3.3) and (3.4) to obtain $\left\|e^{-t\left(A^{2}-\alpha A\right)} \vec{v}\right\|_{E}<\infty$ 
4. Weighted convolution inequalities. The analysis of the nonlinear term will be based on the following convolution theorem due to Kerman [3].

ThEOREM 4.1. Assume that $1<p, q, r<\infty$ and that the following eight conditions hold:

$$
\begin{aligned}
& \text { 1. } \gamma=\alpha+\beta+n\left(\frac{1}{p}+\frac{1}{q}-\frac{1}{r}-1\right) \\
& \text { 2. } \frac{1}{r} \leq \frac{1}{p}+\frac{1}{q} \\
& \text { 3. } \alpha<\frac{n}{p^{\prime}} \\
& \text { 4. } \beta<\frac{n}{q^{\prime}} \\
& \text { 5. } \gamma>-\frac{n}{r} \\
& \text { 6. } \alpha+\beta \geq 0 \\
& \text { 7. } \alpha \geq \gamma \\
& \text { 8. } \beta \geq \gamma
\end{aligned}
$$

Then

$$
\left(\int_{\mathbb{R}^{n}}|f * g(x)|^{r}|x|^{\gamma r} d x\right)^{1 / r} \leq C\left(\int|f(x)|^{p}|x|^{\alpha p}\right)^{1 / p}\left(\int_{\mathbb{R}^{n}}|g(x)|^{q}|x|^{\beta q} d x\right)^{1 / q}
$$

for all measurable $f$ and $g$, where $C$ does not depend on either $f$ or $g$.

Our primary interest is in the case $p=q=r$ and the corresponding inequality

$$
\left(\int_{\mathbb{R}^{n}}|f * g(x)|^{p}|x|^{\gamma p} d x\right)^{1 / p} \leq C\left(\int|f(x)|^{p}|x|^{\alpha p}\right)^{1 / p}\left(\int_{\mathbb{R}^{n}}|g(x)|^{p}|x|^{\beta p} d x\right)^{1 / p}
$$

In this case, condition (2) is superfluous and conditions (7) and (8) are implied by conditions (1), (3), and (4). From now on we denote by $C_{a, b, \ldots}$ a constant whose precise value depends only on $a, b, \ldots$. The preceding remarks are summarized in the following theorem: then

TheOrem 4.2. Let $1<p<\infty$. If $\alpha, \beta<\frac{n}{p^{\prime}}, \alpha+\beta \geq 0$, and $\alpha+\beta>\frac{n}{p^{\prime}}-\frac{n}{p}$,

$$
\begin{aligned}
& \left(\int_{\mathbb{R}^{n}}|f * g(x)|^{p}|x|^{\left(\alpha+\beta-\frac{n}{p^{\prime}}\right) p} d x\right)^{1 / p} \\
& \quad \leq C_{\alpha, \beta, n, p}\left(\int|f(x)|^{p}|x|^{\alpha p}\right)^{1 / p}\left(\int_{\mathbb{R}^{n}}|g(x)|^{p}|x|^{\beta p} d x\right)^{1 / p}
\end{aligned}
$$

for all measurable functions $f$ and $g$.

The convolution of two sequences $\vec{u}, \vec{v} \in V$ is given by

$$
(\vec{u} * \vec{v})_{k}=\sum_{h} u_{k-h} v_{h}
$$

whenever the sum is convergent. Theorem 4.2 will be used to prove the following inequality in weighted sequence spaces.

THEOREM 4.3. Under the same hypotheses as Theorem 4.2 we have that

$$
\|\vec{u} * \vec{v}\|_{\alpha+\beta-\frac{n}{p^{\prime}}, p} \leq C_{\alpha, \beta, n, p}\|\vec{u}\|_{\alpha, p}\|\vec{v}\|_{\beta, p}
$$


for all $\vec{u} \in V_{\alpha, p}$ and $\vec{v} \in V_{\beta, p}$.

Proof. For each $k \in \mathbb{Z}^{n}$ we denote by $Q_{k}$ the open $n$-cube in $\mathbb{R}^{n}$ with edges parallel to the coordinate axes, sidelength one, and center $k$. Two cubes $Q_{k}$ and $Q_{l}$ are said to be adjacent if $|k-l|_{\infty}=1$. First we observe that if $t>-n$ then

$$
\int_{Q_{k}}|x|^{t} d x \approx(1+|k|)^{t} .
$$

If $k \neq 0$, then $|x| \geq C_{n}$ for all $x \in Q_{k}$. In this instance we have

$$
|x| \leq|x-k|+|k| \leq C_{n}(1+|k|)
$$

and

$$
|k| \leq|x-k|+|x| \leq C_{n}|x|,
$$

hence

$$
1+|k| \leq C_{n}|x|
$$

Since

$$
\int_{Q_{k}}(1+|k|)^{t} d x=(1+|k|)^{t}
$$

the equivalence (4.1) is valid for any real $t$. On the other hand, if $k=0$, then

$$
\int_{Q_{0}}|x|^{t} d x=C_{n, t}
$$

provided that $t>-n$, establishing (4.1). Now let us examine the structure of the convolution. Let $\gamma=\alpha+\beta-\frac{n}{p^{\prime}}$. Since $\gamma p>-n$ we have

$$
\begin{aligned}
\|\vec{u} * \vec{v}\|_{\gamma, p}^{p} & =\sum_{k}(1+|k|)^{\gamma p}\left|(\vec{u} * \vec{v})_{k}\right|^{p} \\
& \leq \sum_{k}(1+|k|)^{\gamma p}\left(\sum_{h}\left|u_{h}\right|\left|v_{k-h}\right|\right)^{p} \\
& \lesssim \sum_{k} \int_{Q_{k}}|x|^{\gamma p}\left(\sum_{h}\left|u_{h}\right|\left|v_{k-h}\right|\right)^{p} d x .
\end{aligned}
$$

Define functions $f, g: \mathbb{R}^{n} \rightarrow[0, \infty)$ by

$$
f(x)=\left|u_{k}\right|, \quad g(x)=\left|v_{k}\right|, \quad x \in Q_{k} .
$$

Fix $k \in \mathbb{Z}^{n}$. Then

$$
\sum_{h}\left|u_{h}\right|\left|v_{k-h}\right|=\sum_{h} \int_{Q_{h}} f(y)\left|v_{k-h}\right| d y .
$$

Note that $y \in Q_{h}$ if and only if $k-y \in Q_{k-h}$. Therefore $g(k-y)=\left|v_{k-h}\right|$ for all $y \in Q_{h}$ and

$$
\sum_{h} \int_{Q_{h}} f(y)\left|v_{k-h}\right| d y=\sum_{h} \int_{Q_{h}} f(y) g(k-y) d y .
$$


Now let $x \in Q_{k}$. If $y \in Q_{h}$, then $k-y$ and $x-y$ belong to adjacent $n$-cubes. Thus there exists $j \in \mathbb{Z}^{n}$ with $|j|_{\infty} \leq 1$ such that $g(k-y)=g(x+j-y)$. It follows that

$$
g(k-y) \leq \sum_{|j|_{\infty} \leq 1} g(x+j-y)
$$

and consequently

$$
\sum_{h}\left|u_{h}\right|\left|v_{k-h}\right| \leq \sum_{|j| \leq 1} \int_{\mathbb{R}^{n}} f(y) g(x+j-y) d y, \quad x \in Q_{k} .
$$

Denote by $\tau_{z}$ the translation operator given by $\left(\tau_{z} \psi\right)(y)=\psi(y-z)$, and define

$$
G=\sum_{|j|_{\infty} \leq 1} \tau_{-j} g
$$

Then (4.3) may be written as

$$
\sum_{h}\left|u_{h}\right|\left|v_{k-h}\right| \leq \sum_{|j| \leq 1} \int_{\mathbb{R}^{n}} f(y) \tau_{-j} g(x-y) d y=f * G(x) .
$$

Inequality (4.2), Theorem 4.2, and Minkowski's inequality imply that

$$
\begin{aligned}
\|\vec{u} * \vec{v}\|_{\gamma, p} & \lesssim\left(\int_{\mathbb{R}^{n}}|x|^{\gamma p}(f * G(x))^{p} d x\right)^{1 / p} \\
& \lesssim\left(\int_{\mathbb{R}^{n}}|x|^{\alpha p} f(x)^{p} d x\right)^{1 / p}\left(\int_{\mathbb{R}^{n}}|x|^{\beta p} G(x)^{p} d x\right)^{1 / p} \\
& \lesssim \sum_{|j| \leq 1}\left(\int_{\mathbb{R}^{n}}|x|^{\alpha p} f(x)^{p} d x\right)^{1 / p}\left(\int_{\mathbb{R}^{n}}|x|^{\beta p} \tau_{-j} g(x)^{p} d x\right)^{1 / p}
\end{aligned}
$$

If $|j|_{\infty} \leq 1,(4.1)$ and the definition of $g$ imply that

$$
\begin{aligned}
\int_{\mathbb{R}^{n}}|x|^{\beta p}\left(\tau_{-j} g\right)(x)^{p} d x & \lesssim \sum_{k}(1+|k|)^{\beta p} \int_{Q_{k}}\left(\tau_{-j} g\right)(x)^{p} d x \\
& =\sum_{k}(1+|k|)^{\beta p} \int_{Q_{k-j}} g(x)^{p} d x \\
& =\sum_{k}(1+|k|)^{\beta p}\left|v_{k-j}\right|^{p} \\
& \lesssim \sum_{k}(1+|k|)^{\beta p}\left|v_{k}\right|^{p}
\end{aligned}
$$

so that

$$
\left(\int_{\mathbb{R}^{n}}|x|^{\beta p} \tau_{-j} g(x)^{p} d x\right)^{1 / p} \lesssim\|\vec{v}\|_{\beta, p}
$$

Likewise we have that

$$
\left(\int_{\mathbb{R}^{n}}|x|^{\alpha p} f(x)^{p} d x\right)^{1 / p} \lesssim\|\vec{u}\|_{\alpha, p}
$$


which yields the desired result.

The following corollary extends Theorem 4.3 to three-term convolutions.

COROLlary 4.4. If $\alpha, \beta, \gamma<\frac{n}{p^{\prime}}, \alpha+\beta+\gamma \geq \frac{n}{p^{\prime}}$, and $\alpha+\beta+\gamma>\frac{2 n}{p^{\prime}}-\frac{n}{p}$, then

$$
\|\vec{u} * \vec{v} * \vec{w}\|_{\alpha+\beta+\gamma-\frac{2 n}{p^{\prime}}, p} \leq C_{\alpha, \beta, n, p}\|\vec{u}\|_{\alpha, p}\|\vec{v}\|_{\beta, p}\|\vec{w}\|_{\gamma, p} .
$$

Proof. The stated assumptions imply that

$$
\left(\alpha+\beta-\frac{n}{p^{\prime}}\right), \gamma<\frac{n}{p^{\prime}},\left(\alpha+\beta-\frac{n}{p^{\prime}}\right)+\gamma \geq 0, \text { and }\left(\alpha+\beta-\frac{n}{p^{\prime}}\right)+\gamma>\frac{n}{p^{\prime}}-\frac{n}{p}
$$

so that Theorem 4.3 implies

$$
\begin{aligned}
\|\vec{u} * \vec{v} * \vec{w}\|_{\alpha+\beta+\gamma-\frac{2 n}{p^{\prime}}, p} & =\|(\vec{u} * \vec{v}) * \vec{w}\|_{\left(\alpha+\beta-\frac{n}{p^{\prime}}\right)+\gamma-\frac{n}{p^{\prime}}, p} \\
& \leq C_{\alpha, \beta, n, p}\|\vec{u} * \vec{v}\|_{\alpha+\beta-\frac{n}{p^{\prime}}, p}\|\vec{w}\|_{\gamma, p} .
\end{aligned}
$$

Finally note that the hypotheses imply $\alpha+\beta \geq 0$ and $\alpha+\beta>\frac{n}{p^{\prime}}-\frac{n}{p}$ and apply Theorem 4.3 again.

The following simplification to Corollary 4.4 will be used in the sequel.

COROLlary 4.5. If $\frac{n}{3 p^{\prime}}<\alpha<\frac{n}{p^{\prime}}$ and $\alpha>\frac{2 n}{3 p^{\prime}}-\frac{n}{3 p}$, then

$$
\|\vec{u} * \vec{v} * \vec{w}\|_{3 \alpha-\frac{2 n}{p^{\prime}}, p} \leq C_{\alpha, \beta, n, p}\|\vec{u}\|_{\alpha, p}\|\vec{v}\|_{\alpha, p}\|\vec{w}\|_{\alpha, p} .
$$

\section{Estimate on the nonlinear term.}

Proposition 5.1. The mapping $S$ defined by (2.4) is a bounded mapping from $E \times E \times E$ to $E$.

Proof. We will make a general estimate of the term $\|S(\vec{u}, \vec{v}, \vec{w})\|_{\delta, p}$ for $\delta \in \mathbb{R}$ satisfying

$$
0<\delta+2-3 \theta-3 \gamma+\frac{2 n}{p^{\prime}}<4
$$

The restrictions placed on $\gamma$ in (2.3) imply that both $\theta, \theta+\gamma$ are admissible values of $\delta$ satisfying (5.1). Let $\vec{u}(\cdot), \vec{v}(\cdot), \vec{w}(\cdot) \in E$. The triangle inequality implies

$$
\|S(\vec{u}, \vec{v}, \vec{w})(t)\|_{\lambda t, \delta, p} \leq \beta \int_{0}^{t}\left\|e^{-(t-s)\left(A^{2}-\alpha A\right)} B[\vec{u}(s), \vec{v}(s), \vec{w}(s)]\right\|_{\lambda t, \delta, p} d s .
$$

For simplicity of notation we will suppress the variable $s$ from the trilinear term. Let $0<s<t$. Employing (3.1) above we have

$$
\begin{aligned}
\left\|e^{-(t-s)\left(A^{2}-\alpha A\right)} B[\vec{u}, \vec{v}, \vec{w}]\right\|_{\lambda t, \delta, p}^{p} & \leq \sum_{k} e^{\lambda p t|k|} e^{-p(t-s)\left(|k|^{4}-\alpha|k|^{2}\right)}|k|^{\delta p}\left|B[\vec{u}, \vec{v}, \vec{w}]_{k}\right|^{p} \\
& =\sum_{k} e^{\lambda p s|k|} e^{-p(t-s)\left(|k|^{4}-\alpha|k|^{2}-\lambda|k|\right)}|k|^{\delta p}\left|B[\vec{u}, \vec{v}, \vec{w}]_{k}\right|^{p} \\
& \leq e^{p \kappa(t-s)} \sum_{k} e^{\lambda p s|k|} e^{-\frac{1}{2} p(t-s)|k|^{4}}|k|^{\delta p}\left|B[\vec{u}, \vec{v}, \vec{w}]_{k}\right|^{p} \\
& \leq e^{p \kappa(t-s)} \sum_{k} e^{\lambda p s|k|} e^{-\frac{1}{2} p(t-s)|k|^{4}}|k|^{(\delta+2) p}(|\vec{u}| *|\vec{v}| *|\vec{w}|)_{k}^{p}
\end{aligned}
$$


where we used (1.4) on the last line. An application of (3.2) and (5.1) yields

$$
e^{-\frac{1}{2} p(t-s)|k|^{4}}|k|^{(\delta+2) p} \leq C_{\delta, \gamma, \theta, n, p}(t-s)^{-\frac{p}{4}\left(\delta+2-3 \theta-3 \gamma+\frac{2 n}{p^{\prime}}\right)}|k|^{\left(3 \theta+3 \gamma-\frac{2 n}{p^{\prime}}\right) p}
$$

so that

$$
\begin{aligned}
& \left\|e^{-(t-s)\left(A^{2}-\alpha A\right)} B[\vec{u}, \vec{v}, \vec{w}]\right\|_{\lambda t, \delta, p} \\
& \quad \leq C_{\delta, \gamma, \theta, n, p} e^{\kappa(t-s)}(t-s)^{-\frac{1}{4}\left(\delta+2-3 \theta-3 \gamma+\frac{2 n}{p^{\prime}}\right)}\||\vec{u}| *|\vec{v}| *|\vec{w}|\|_{\lambda s, 3 \theta+3 \gamma-\frac{2 n}{p^{\prime}}, p} .
\end{aligned}
$$

The definition $\theta=\frac{n}{p^{\prime}}-1$ and assumption (2.3) imply

$$
\frac{n}{3 p^{\prime}}<\theta+\gamma<\frac{n}{p^{\prime}} \text { and } \quad \theta+\gamma>\frac{2 n}{3 p^{\prime}}-\frac{n}{3 p} .
$$

Since

$$
e^{\lambda s|k|}(|\vec{u}| *|\vec{v}| *|\vec{w}|)_{k} \leq \sum_{h} \sum_{j} e^{\lambda s|k-h-j|}\left|u_{k-h-j}\right| e^{\lambda s h}\left|v_{h}\right| e^{\lambda s j}\left|w_{j}\right|,
$$

we may apply Corollary 4.5 to obtain

$$
\||\vec{u}| *|\vec{v}| *|\vec{w}|\|_{\lambda s, 3 \theta+3 \gamma-\frac{2 n}{p^{\prime}, p}} \leq C_{\gamma, \theta, n, p}\|\vec{u}\|_{\lambda s, \theta+\gamma, p}\|\vec{v}\|_{\theta+\gamma, p}\|\vec{w}\|_{\lambda s, \theta+\gamma, p} .
$$

We arrive at the estimate

$$
\begin{aligned}
& \|S(\vec{u}, \vec{v}, \vec{w})(t)\|_{\delta, p} \\
& \leq C_{\delta, \gamma, \theta, n, p} e^{\kappa t} \beta \int_{0}^{t}(t-s)^{-\frac{1}{4}\left(\delta+2-3 \theta-3 \gamma+\frac{2 n}{p^{\prime}}\right)}\|\vec{u}\|_{\lambda s, \theta+\gamma, p}\|\vec{v}\|_{\lambda s, \theta+\gamma, p}\|\vec{w}\|_{\lambda s, \theta+\gamma, p} d s .
\end{aligned}
$$

At this point it is convenient to denote, for $\vec{u}(\cdot) \in E$,

$$
\|\vec{u}(\cdot)\|_{E^{\prime}}=\sup _{0<t \leq T}\|\vec{u}(t)\|_{\lambda t, \theta+\gamma, p}
$$

so that $s^{\frac{\gamma}{4}}\|\vec{u}(s)\|_{\lambda s, \theta+\gamma, p} \leq\|\vec{u}(\cdot)\|_{E^{\prime}}$ for all $0<s \leq T$. This leads to the estimate

$$
\|S(\vec{u}, \vec{v}, \vec{w})(t)\|_{\delta, p} \leq C_{\delta, \gamma, \theta, n, p} e^{\kappa t} \beta\|\vec{u}\|_{E^{\prime}}\|\vec{v}\|_{E^{\prime}}\|\vec{w}\|_{E^{\prime}} \int_{0}^{t}(t-s)^{-\frac{1}{4}\left(\delta+2-3 \theta-3 \gamma+\frac{2 n}{p^{\prime}}\right)} s^{-\frac{3 \gamma}{4}} d s .
$$

Since

$$
\frac{1}{4}\left(\delta+2-3 \theta-3 \gamma+\frac{2 n}{p^{\prime}}\right)<1 \quad \text { and } \quad \frac{3 \gamma}{4}<1
$$

this integral equals a constant $C_{\delta, \theta, \gamma, n, p}$ times

$$
t^{1-\frac{1}{4}\left(\delta+2-3 \theta-3 \gamma+\frac{2 n}{p^{\prime}}\right)-\frac{3 \gamma}{4}}=t^{\frac{1}{4}\left(2-\delta+3 \theta-\frac{2 n}{p^{\prime}}\right)} .
$$

We conclude there exists a constant $C=C_{\delta, \gamma, \theta, n, p}$ with the property that

$$
\|S(\vec{u}, \vec{v}, \vec{w})(t)\|_{\delta, p} \leq C e^{\kappa T} \beta t^{\frac{1}{4}\left(2-\delta+3 \theta-\frac{2 n}{p^{\prime}}\right)}\|\vec{u}\|_{E^{\prime}}\|\vec{v}\|_{E^{\prime}}\|\vec{w}\|_{E^{\prime}}
$$

for all $0<t \leq T$. When $\delta=\theta$ this gives

$$
\|S(\vec{u}, \vec{v}, \vec{w})(t)\|_{\theta, p} \leq C \beta t^{\frac{1}{4}\left(2+2 \theta-\frac{2 n}{p^{\prime}}\right)}\|\vec{u}\|_{E^{\prime}}\|\vec{v}\|_{E^{\prime}}\|\vec{w}\|_{E^{\prime}}
$$


and when $\delta=\theta+\gamma$ this gives

$$
\|S(\vec{u}, \vec{v}, \vec{w})(t)\|_{\theta+\gamma, p} \leq C \beta t^{\frac{1}{4}\left(2+2 \theta-\gamma-\frac{2 n}{p^{\prime}}\right)}\|\vec{u}\|_{E^{\prime}}\|\vec{v}\|_{E^{\prime}}\|\vec{w}\|_{E^{\prime}}
$$

so that

$$
t^{\frac{\gamma}{4}}\|S(\vec{u}, \vec{v}, \vec{w})(t)\|_{\theta+\gamma, p} \leq C \beta t^{\frac{1}{4}\left(2+2 \theta-\frac{2 n}{p^{\prime}}\right)}\|\vec{u}\|_{E^{\prime}}\|\vec{v}\|_{E^{\prime}}\|\vec{w}\|_{E^{\prime}} .
$$

Since $\theta=\frac{n}{p^{\prime}}-1$ we conclude

$$
\|S(\vec{u}, \vec{v}, \vec{w})(\cdot)\|_{E} \leq C_{\alpha, n, p} \beta\|\vec{u}\|_{E^{\prime}}\|\vec{v}\|_{E^{\prime}}\|\vec{w}\|_{E^{\prime}} .
$$

It follows that $S$ is a bounded mapping from $E \times E \times E$ to $E$.

6. Construction of the local solution. Write $E=E_{T}$ to emphasize the dependence of the space $E$ on $T$. Let $u_{0} \in \dot{V}_{\theta, p}$ and write $\vec{v}(t)=e^{-t\left(A^{2}-\alpha A\right)} \vec{u}_{0}$. Define

$$
B=\left\{\vec{u}(\cdot) \in E:\|\vec{u}(\cdot)-\vec{v}(\cdot)\|_{E_{T}^{\prime}} \leq\|\vec{v}(\cdot)\|_{E_{T}^{\prime}}\right\}
$$

and define $L: E \rightarrow E$ by

$$
L \vec{u}(t)=\vec{v}(t)+S(\vec{u}, \vec{u}, \vec{u})(t) .
$$

Let $C$ be the constant from (5.3). It follows that

$$
\|L \vec{u}(\cdot)-\vec{v}(\cdot)\|_{E_{T}^{\prime}} \leq C \beta\|\vec{u}(\cdot)\|_{E_{T}^{\prime}}^{3} \leq 8 C \beta\|\vec{v}(\cdot)\|_{E_{T}^{\prime}}^{3}
$$

for all $\vec{u}(\cdot) \in B$. The trilinearity of $S$ implies

$S\left(\vec{u}_{1}, \vec{u}_{1}, \vec{u}_{1}\right)-S\left(\vec{u}_{2}, \vec{u}_{2}, \vec{u}_{2}\right)=S\left(\vec{u}_{1}-\vec{u}_{2}, \vec{u}_{2}, \vec{u}_{2}\right)+S\left(\vec{u}_{1}, \vec{u}_{1}-\vec{u}_{2}, \vec{u}_{2}\right)+S\left(\vec{u}_{1}, \vec{u}_{1}, \vec{u}_{1}-\vec{u}_{2}\right)$

so that

$$
\begin{aligned}
\left\|L \vec{u}_{1}(\cdot)-L \vec{u}_{2}(\cdot)\right\|_{E_{T}^{\prime}} & \leq\left\|S\left(\vec{u}_{1}(\cdot), \vec{u}_{1}(\cdot), \vec{u}_{1}(\cdot)\right)-S\left(\vec{u}_{2}(\cdot), \vec{u}_{2}(\cdot), \vec{u}_{2}(\cdot)\right)\right\|_{E_{T}^{\prime}} \\
& \leq 12 C \beta\left\|\vec{u}_{1}(\cdot)-\vec{u}_{2}(\cdot)\right\|_{E_{T}^{\prime}}\|\vec{v}(\cdot)\|_{E_{T}^{\prime}}^{2} .
\end{aligned}
$$

Equations (6.1) and (6.2) show that $L: B \rightarrow B$ is a contraction provided that $\beta$ is sufficiently small. The Banach fixed point theorem provides a solution $\vec{u}(\cdot) \in B$ to the equation

$$
\vec{u}(\cdot)=L \vec{u}(\cdot)
$$

This fixed point satisfies

$$
\vec{u}(t)=\vec{v}(t)+S(\vec{u}, \vec{u}, \vec{u})(t), \quad 0 \leq t \leq T .
$$

which is precisely a solution to (2.1).

On the other hand, for any $\vec{w} \in \dot{V}$ having most finitely many nonzero terms we have $\vec{w} \in \dot{V}_{\theta+\gamma, p}$ and thus

$$
\begin{aligned}
t^{\frac{\gamma}{4}}\left\|e^{-t\left(A^{2}-\alpha A\right)} \vec{u}_{0}\right\|_{\lambda t, \theta+\gamma, p} & \leq t^{\frac{\gamma}{4}}\left\|e^{-t\left(A^{2}-\alpha A\right)}\left(\vec{u}_{0}-\vec{w}\right)\right\|_{\lambda t, \theta+\gamma, p}+t^{\frac{\gamma}{4}}\left\|e^{-t\left(A^{2}-\alpha A\right)} \vec{w}\right\|_{\lambda t, \theta+\gamma, p} \\
& \leq C e^{\kappa T}\left\|\vec{u}_{0}-\vec{w}\right\|_{\theta, p}+t^{\frac{\gamma}{4}} e^{\kappa T}\|\vec{w}\|_{\theta+\gamma, p}
\end{aligned}
$$


for all $0<t \leq T$. Since $\|\vec{v}-\vec{w}\|_{\theta, p}$ can be made arbitrarily small we conclude that

$$
\lim _{t \rightarrow 0^{+}} t^{\frac{\gamma}{4}}\left\|e^{-t\left(A^{2}-\alpha A\right)} \vec{u}_{0}\right\|_{\lambda t, \theta+\gamma, p}=0
$$

This implies

$$
\lim _{T \rightarrow 0^{+}}\|\vec{v}(\cdot)\|_{E_{T}^{\prime}}=0
$$

so for arbitrary $\beta>0$ equations (6.1) and (6.2) show that $L: B \rightarrow B$ is a contraction provided that $T$ is sufficiently small. As above we obtain a fixed point of $L$ satisfying (6.3), completing the argument.

\section{REFERENCES}

[1] A. Biswas AND H. BAE, Gevrey regularity for a class of dissipatice equations with analytic nonlinearity, preprint.

[2] A. Biswas And D. Swanson, Gevrey regularity of solutions to the 3D Navier-Stokes equations, Fluids and Waves, pp. 83-90, Contemp. Math., 440, Amer. Math. Soc., Providence, RI, 2007.

[3] R. A. Kerman, Convolution theorems with weights, Trans. Amer. Math. Soc., 280:1 (1983), pp. 207-219.

[4] G. Sell and Y. You, Dynamics of Evolutionary Equations, Applied Mathematical Sciences 143, Springer, 2002.

[5] R. Temam, Infinite-Dimensional Dynamical Systems in Mechanics and Physics, 2nd Ed., Applied Mathematical Sciences 68, Springer, 1997. 